\title{
Measuring player creativity in digital entertainment games using the Creativity in Gaming Scale
}

\author{
Johanna Hall, Christothea Herodotou and \\ loanna lacovides
}

\section{I2.I Introduction}

In 2020 , the global population of gamers stood at 2.7 billion with an increase of over $6 \%$ globally from the previous year. Breaking this down further, those who play games have increased in all regions of the world, with the largest increase of gamers being in the Middle East and Africa, with a $14 \%$ increase from the previous year. This growth is even more prominent with the rise of smartphone usage and which is now the largest gaming platform in global terms (Newzoo, 2020). The increasing ubiquity of digital games, now widely available on a variety of different mediums including mobile, PC, console and streaming services, has meant that the benefits of gaming are reaching an even wider, more diverse demographic.

Digital games have been argued to be an effective medium to foster creativity as they encourage players to overcome challenging problems and actively experiment with different ideas and solutions free from external constraints (Leng et al., 2010). Games often comprise ill-structured challenges (e.g., problems with more than one solution) which have been argued to facilitate intrinsically motivating flow experiences leading to creativity (Kiili, 2005). The relationship between creativity and gameplay has been investigated by studies such as Moffat et al. (2017) who found that playing games contributes to a creative state of mind, and Blanco-Herrera et al. (2019) who identified a positive correlation between gameplay and trait creativity scores.

While general conceptualisations of creativity attempt to account for the many facets of, what has been argued to be, a multidimensional construct (Shute \& Wang, 2016), they do not provide information on how creativity may manifest specifically within digital games. By understanding these unique manifestations of creativity, digital games may be used as an alternative, widely available tools for open-world learning. In this way, not only educators may select different games to develop creativity in students, but learners will have the autonomy to identify areas of creativity they wish to develop and select games according to their preferences. 


\section{I2.I.I Creativity in digital games}

Hall et al. (2020a) attempted to classify the different ways creativity is expressed in games by identifying three main categories: creativity as problem-solving, creativity as appropriation and creativity as affective change. Creativity as problem-solving relates to the unique solutions and approaches to gaming problems that players create, and is likened to "Little C" (Kaufman \& Beghetto, 2009) or "Everyday creativity" (Richards, 2007) in that the creative solutions/ideas are unique to the individual who creates them. Work such as Kiili’s (2005) Experiential Gaming Model extrapolated how games facilitate creative problem-solving through an experiential process where players constantly testing, reflecting on, and refining solutions. Similarly, Iacovides et al. (2014) illustrated the unique approaches players create to navigating gameplay breakdowns, such as taking a break or using a trial and error approach.

Creativity as appropriation relates to the ways players go above and beyond what developers expect, personalising the gameplay experience (Herodotou et al., 2012). Appropriation includes emergent (Jarrett, 2014) and transgressive play (Aarseth, 2007) where players can combine game variables and mechanics in unintended ways, use glitches and create additional challenges such as speedrunning (i.e., completing the game in as short a time as possible). Furthermore, appropriation also includes transformative play (Sotamaa, 2007) including the creation and use of modifications, and user-created content (Burri, 2011) within the game such as object creation, and around the game such as fanfiction and fanart.

Creativity as affective change is most synonymous with Kaufman and Beghetto's (2009) "Mini C" whereby creativity is conceptualised as a player's unique interpretation of gameplay and game narrative which can lead to "reflection on the game's narrative, and as a result, the alteration of thought patterns, beliefs and perspectives" (Hall et al., 2020a, p. 9). Work such as Bopp et al. (2018) has investigated how games can instigate affective change, including reflection (Mekler et al., 2018), through emotional challenges which are presented through choice-based dialogues and ambiguous narratives, and perspective-challenging moments where players' prior concepts, attitudes and expectations are challenged (Whitby et al., 2019).

\subsubsection{Measures of creativity}

Creativity has been argued to be a hard-to-measure construct due to a variety of factors, such as a lack of clear definition and operationalisation, theoretical multidimensionality, and difficulty separating trait from state (e.g., some people may only be creative in certain situations, while others may be generally creative) (Shute \& Wang, 2016). As such, there exist a number of metrics that aim to assess creativity from a variety of perspectives.

For example, some psychometric assessments rely on divergent thinking which has been "conceptualised as one component of creative thinking [and] remains an important concept among creativity researchers" (Baer, 2016, p. 9). The most wellknown divergent thinking test is the Torrance Tests of Creative Thinking (TTCT) (Torrance, 1966) which measures creative attributes such as fluency (total number 
of ideas), flexibility (number of different categories or kinds of ideas), originality (distinctiveness of an idea), and elaboration (richness of detail and broadness of scope of ideas) (Baer, 2016). However, while widely used, TTCT tests have been criticised for measuring only one aspect of a multi-faceted construct, and being too closely linked to intelligence (Lubart, 1994). Other psychometric approaches such as the Remote Association Test (RAT) (Mednick, 1968) assess an individual's ability to form associations and combinations between diverse knowledge elements, and involves a series of three words indirectly related to each other which the participant must connect with a fourth word (Lubart, 1994). The RAT has been criticised for having poor content validity by measuring verbal ability and academic performance instead of creativity (Lubart, 1994).

Other metrics aim to measure creativity based on personality factors such as the Creative Personality Scale (CPS) (Gough, 1979) and the Creativity Style Questionnaire (Kumar et al., 1997). Biographical inventories assess creative talent via observational measures by instructors, supervisors, or classmates to assess behaviour and compare it to creative outputs and sensitivity such as the Creative Behavior Inventory (CBI) (Kirschenbaum, 1989). Measurements of creative products include metrics such as the Creative Product Assessment Matrix (Besemer \& O'Quin, 1987) which attempts to analyse how creative a product/idea is.

While far less common, there are some examples of measurements of creativity for digital games. For example, Chuang et al. (2015) documented the creation of an objective metric for measuring creativity in the game Arctic Quest 2. Using a computerised fuzzy logic system that extended evaluations beyond true/false and good/bad, player's trophies and points were recorded and used to calculate scores on fluency, flexibility, elaboration and sensibility. While Chuang et al's (2015) computerised method eliminated some of the subjectivity in creativity assessment, it does not measure originality (e.g., novelty and relevance of ideas) which has been argued to be a key component of creativity (Runco \& Jaeger, 2012). Furthermore, it is based on only one game, and does not account for the specific manifestations of creativity in digital games, instead of using the psychometric properties of general creativity metrics.

Huang and Chuang (2012) devised the Creativity Assessment Scale of Digital Game Story Design (CAS-DGSD) to measure the creativity involved in creating game narrative. The CAS-DGSD includes the creativity dimensions of novelty, complexity, imagination, variation and application, and the digital game story constructs of content, narrative device, organisation, word choice and game feature. While the CAS-DGSD provides a valuable assessment tool for evaluating game narrative, it is geared more towards the creativity involved in designing a game, rather than the creativity involved in playing one.

In essence, a range of metrics exist to measure creativity from a variety of theoretical standpoints; however, the majority of these do not pertain to the unique expressions of creativity in digital games. The scope of creative practices which games facilitate may be difficult to quantify with traditional measures of creativity such as the TTCT (Torrance, 1993) and the CPS (Gough, 1979), and work which has attempted to create metrics for the assessment of creativity in digital games 
such as Chuang et al.s (2015) fuzzy logic system focused on one game genre using existing psychometric constructs to measure creativity. As such, this chapter details the development of a scale to measure creativity unique to digital games and is not based on existing psychometric or traditional measures. The scale operationalises creativity specific to digital games and provides a measure of the unique expressions of creativity and associated constructs from the ground up.

\section{2.2 Method}

\section{2.2. I Research instrument}

The instrument for data collection was an online survey, which consisted of three parts with the first comprising the consent questionnaire. The second part asked respondents about their current gaming habits, including how many hours a week they spent playing online and offline games, genre of games played, format of gaming (e.g., PC, console, handheld) and what type of gamer they identified as, if any (e.g., casual, moderate, hardcore). The third part of the survey comprised a total of 71 attitude statements across seven sections. The statements related to themes developed from a previous qualitative study (discussed in Hall et al., 2020a, 2020b) which identified three unique expressions of creativity in digital games using a thematic approach; problem-solving, appropriation and affective change, in addition to further related themes including player conceptualisations of creativity, design affordances for creativity, learning from creativity and transferability of creativity. As such, three sections related to the different forms of creative expression, one related to how players conceptualised creativity, one related to learning outcomes from creativity, one related to the transferability of game-based creativity and one related to creative design affordances in games. Scale items were on a standard 5-point Likert scale, with 1 being strongly disagree and 5 being strongly agree. At least one negatively worded item was included in every Likert section.

\subsubsection{Recruitment}

The study advert was posted on a variety of online forums, social media and Reddit. A wide selection of gaming forums was chosen to capture multiple different game genres and, when available, the advert was placed in sub-forums such as off-topic (non-game related) and community creation areas. Forums included the popular platform Steam, in addition to game-specific forums such as Kerbal Space Program and Bioware. Adverts were also placed on speedrunning and game modification forums such as Nexus Mods. Reddit was used to target specific games from a variety of genres (e.g., r/ggoverwatch, r/witcher3) and groups (e.g., r/ gamedev, r/gamingpc) and the survey specific sub-reddit $\mathrm{r}$ /samplesize was also used. The advert was posted on the principal researcher's Facebook and was shared on Twitter using hashtags such as \#videogamers and \#gamedevs. Due to the high proportion of males in the sample, Twitter was also used to target female gaming and tech groups such as \#womenintech and \#girlgamers. 


\subsubsection{Participants and data analysis}

A total of 251 respondents completed the survey, comprising 160 males, 82 females, 6 nonbinary, and 3 undisclosed. The most common age bracket was 25-34 $(n=$ 108), followed by $18-24(n=89)$. The sample consisted of predominantly American and European nationalities and was fairly even across education levels, with the most common being undergraduate and college (high school $=39$, college $=60$, undergraduate $=81$, postgraduate $=40$, doctorate $=18$, and other $=10$ ) .

The stage of analysis comprised dimension reduction using exploratory Principal Component Analysis (PCA) with Varimax rotation in order to "summarize data so that relationships and patterns can be easily understood [and] to regroup variables into a limited set of clusters based on shared variance"(Gie Yong \& Pearce, 2013, p. 79). The higher cut-off point of. 45 which is described as fair to good was adopted (Tabachnick \& Fidell, 2007). All of cross-loading items were also removed.

\section{2.3 Results}

\section{2.3. I Descriptive statistics}

Respondents played a wide variety of games with the most common online genres being massively multiplayer online role-playing games (MMORPG) $(n=127)$ and massively multiplayer online first-person shooters (MMOFPS) $(n=106)$, and the most common offline game genres being role playing $(n=214)$, action/adventure $(n=187)$, strategy/tactics $(n=146)$ and first-person shooter $(n=124)$. The mean hours spent playing online games a week was $12.2(\mathrm{SD}=14.4)$ and the mean hours spent per week playing offline games was $14.0(\mathrm{SD}=12.3)$. The mean gaming session lasted $3.4(\mathrm{SD}=2.3$ ) hours. The most common gaming medium was PC/ Mac/Linux $(n=222)$ followed by Console $(n=133)$.

\subsubsection{Factor analysis}

Items were subjected to PCA using an iterative process whereby all items which loaded under . 45 and cross-loaded were removed before PCA was rerun until no items cross-loaded or loaded under .45. The resulting initial analysis of the 71 scale items produced a twenty-factor model accounting for $68 \%$ of variance, with a Kaiser-Meyer-Olkin value of .814 and a significant result for Bartlett's test of Sphericity $\left(X^{2}=7555.407, \mathrm{DF}=2485, p<0.001\right)$ indicating that a reasonable factor solution could be computed. Upon analysis of the scree plot, it was evident that five- and six-factor solutions could be supported (Figure 12.1).

Both five- and six-factor solutions were attempted, with the difference of variance between them being $1 \%$ (55\% and 56\%, respectively). Due to the minimal variance difference and due to the five-factor solution providing ease of identification of the constructs within the data, it was selected as the model of choice.

The five-factor model was subjected to the same iterative process as the original factor model whereby all items with loadings under .45 and cross-loadings were removed and PCA was rerun until no items cross-loaded or loaded under.45. The 


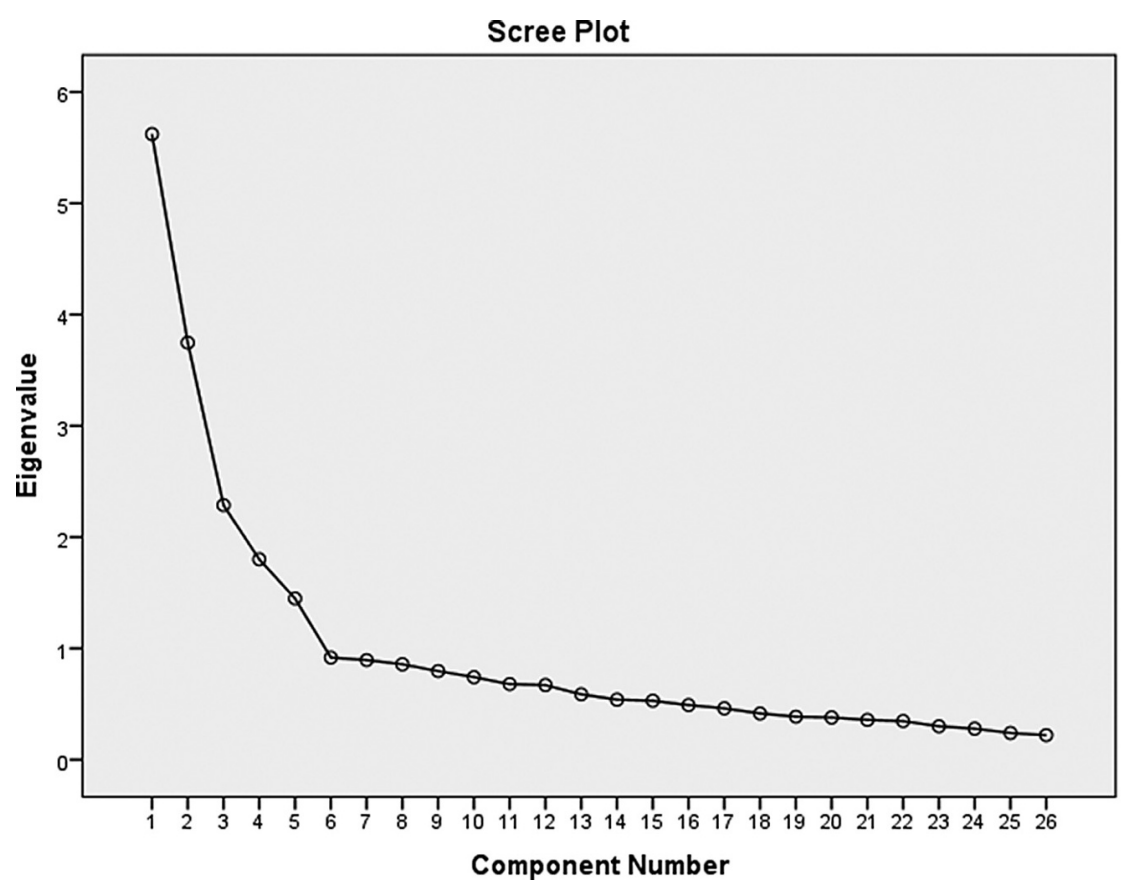

Figure 12.I Scree plot of initial twenty-factor mode.

resulting model accounted for $57.4 \%$ of variance. The Kaiser-Meyer-Olkin measure of sampling adequacy was good at.835 and Barlett's Test of Sphericity was significant $\left(X^{2}=2303.942, \mathrm{DF}=325, p=.000\right)$. Determinant was above the acceptable 0.0001. The instrument was named the Creativity in Gaming Scale (CGS) (see Table 12.1 for factor item loadings and Table 12.2 for variances, means, standard deviations and alpha).

The first factor accounted for the largest variance in the model at $16.3 \%$ and comprised eight items. Two items were aligned with from the creativity as affective change subscale, two items from the learning subscale and four items from the transferability subscale. All items related to the general use of creativity or creative skills such as problem-solving in other areas of life, except for one item (27.7 I have used what I have learnt from games in my job/workplace) which related to the use of these skills within the domain-specific context of the workplace. As such, the factor was named transferability. The second factor accounted for $13.7 \%$ of variance and included five items from the creativity as appropriation subscale. As such, the factor was named appropriation as all items related to how players could go above and beyond what developers expect. The third factor accounted for $11.3 \%$ of variance and comprised five items with the majority coming from the creativity as problem-solving subscale. The remaining two items came from the design affordances and appropriation subscales. As all items suggested an overall alignment 
Table 12.I Creativity in Gaming Scale

\begin{tabular}{|c|c|c|c|c|c|}
\hline Item & Transferability & Appropriation & Problem-Solving & $\begin{array}{l}\text { Affective } \\
\text { change }\end{array}$ & $\begin{array}{l}\text { Design } \\
\text { Affordances }\end{array}$ \\
\hline $\begin{array}{l}27.7 \text { I have used what I have learnt from games in my } \\
\text { job/workplace }\end{array}$ & .757 & & & & \\
\hline $\begin{array}{l}27.6 \text { Games have influenced my attitudes or behaviours } \\
\text { in other areas of life }\end{array}$ & .745 & & & & \\
\hline $\begin{array}{l}\text { 25.I Playing games has made me come to view things in } \\
\text { everyday life differently }\end{array}$ & .729 & & & & \\
\hline $\begin{array}{l}27.4 \text { Being creative in games gives me a new } \\
\text { perspective on problems and challenges in my } \\
\text { everyday life }\end{array}$ & .722 & & & & \\
\hline $\begin{array}{l}\text { 26.6 Being creative in games has developed my } \\
\text { problem-solving and thinking skills }\end{array}$ & .708 & & & & \\
\hline $\begin{array}{l}25.3 \text { Playing games has made me realise things about } \\
\text { myself }\end{array}$ & .680 & & & & \\
\hline $\begin{array}{l}27.10 \text { I have used the skills and knowledge developed in } \\
\text { games elsewhere in my life }\end{array}$ & .657 & & & & \\
\hline $\begin{array}{l}26.2 \text { I have developed IT/technical skills through being } \\
\text { creative in games }\end{array}$ & .581 & & & & \\
\hline $\begin{array}{l}24.2 \text { I actively seek out glitches } \\
24.5 \text { I enjoy using the game mechanics in new, } \\
\text { unintended ways }\end{array}$ & & $\begin{array}{l}.781 \\
.779\end{array}$ & & & \\
\hline 24.31 try and find shortcuts in games & & .747 & & & \\
\hline $\begin{array}{l}24.4 \text { I use glitches to enable me to progress in the } \\
\text { game }\end{array}$ & & .743 & & & \\
\hline
\end{tabular}


Table I2.I (Continued)

\begin{tabular}{llll}
\hline Item & Transferability & Appropriation & Problem-Solving $\begin{array}{l}\text { Affective } \\
\text { change }\end{array}$ Design \\
Affordances
\end{tabular}

24. I I like to test the boundaries of what the game

.703

allows

24. $10 \mathrm{I}$ try and find ways to adapt and bend the rules of

the game

23. I I enjoy coming up with new strategies when I play games

24.8 I enjoy creating additional challenges for myself in games such as upping the difficulty

23.5 I highly value the sense of achievement I get when overcoming difficult challenges in games

23.6 I enjoy experimenting with what I can do using different game variables

22. I I enjoy games that allow me to try out different play styles

25.5 The narrative of a game is important to me

25.10 I enjoy games which make me question things

25.6 I enjoy games that give me a new perspective of other cultures and societies

22.7 I prefer games that let me choose the personality of my character through dialogue choices

22.6 Games that allow more freedom for the player are more likely to involve creativity

20.8 Games that allow the freedom to build and make things are the most creative

22. 10 Games that allow opportunities to interact with the environment help me be more creative in how I play 
Table 12.2 Factor variances, means, and standard deviations

\begin{tabular}{lccccc}
\hline Factor & No. Items & Variance \% & M & SD & Cronbach's Alpha \\
\hline I: Transferability & 8 & 16.3 & 3.8 & .83 & .86 \\
2: Appropriation & 6 & 13.7 & 3.3 & .75 & .86 \\
3: Problem-solving & 5 & 11.3 & 4.2 & .69 & .76 \\
4: Affective change & 4 & 8.9 & 4.3 & .60 & .62 \\
5: Design affordances & 3 & 7.2 & 4.0 & .77 & .60 \\
Whole Scale & $\mathbf{2 6}$ & $\mathbf{5 7 . 4}$ & $\mathbf{3 . 8}$ &. $\mathbf{3 5}$ & $\mathbf{. 8 2}$ \\
\hline
\end{tabular}

with problem-solving the factor was named problem-solving. The fifth factor comprised four items accounting for $8.9 \%$ of total variance. Three of the four items came from the creativity as affective change subscale, and one from the design affordances subscale relating to dialogue choices (22.7: I prefer games that let me choose the personality of my character through dialogue choices).

Overall, the items aligned with how game narrative can cause players to question or reflect on aspects of life, and as such the factor was named affective change. The final factor accounted for $7.2 \%$ of variance with three items, two of which came from the design affordances subscale. The remaining item came from the player conceptualisations subscale which was aligned with the view that creativity was facilitated by opportunities for object construction (20.8: Games which allow the freedom to build and make things are the most creative). Taken together the items pointed to an alignment with games that facilitated creativity through environmental interaction and object construction, and as such, the factor was named design affordances.

\subsubsection{Reliability analysis}

Cronbach's alpha was computed for each subscale individually and the scale as a whole. Transferability, appropriation and problem-solving produced values of .86, .86 and .76 which are deemed good (Tavakol \& Dennick, 2011). Affective change and design affordances had lower values of.62 and.60, respectively; however, these values may be attributed to the fact that these two factors had lower numbers of items which can reduce the alpha value. Alpha for the scale as a whole was good at.82, indicating a high level of internal consistency.

\section{2.4 Discussion and moving forwards}

The CGS comprised five subscales, three of which aligned to the different expressions of creativity; problem-solving, appropriation and affective change, and two relating to transferability and design affordances, respectively. Affective change $(M=4.3$, $\mathrm{SD}=.60)$ and problem-solving $(M=4.2, \mathrm{SD}=.69)$ had the highest mean scores, suggesting participants had a positive attitude towards games which supported these forms of creative expression. Previous work by Cole et al. (2015) found that narrative was a core component of the gaming experience which may explain the high mean score for affective change. The majority of games include problem-solving elements in the form of ill-structured challenges (Kiili, 2005) which players can 
solve in multiple ways, and due to the ubiquitous nature of these challenges, respondents may have been more likely to recognise the statements in the problemsolving subscale and hence, score higher on this subscale. Appropriation $(M=3.3, \mathrm{SD}$ $=.75$ ) on the other hand had a lower mean score, suggesting a more neutral valence towards activities associated with creativity as appropriation. As previous work found that some players felt glitches detracted them from the gaming experience, or were worried about corrupting their saved data (Hall et al., 2020a), two statements regarding glitches (24.2, I actively seek out glitches, 24.4: I use glitches to enable me to progress in the game) both with lower mean scores $(M<2.5)$ may have impacted on the overall mean score for this subscale.

Respondents scored fairly high on the design affordances subscale $(M=4, \mathrm{SD}=.77)$, suggesting that games which provided greater player freedom, opportunities for constructing objects, and supporting interaction with the environment were important for cultivating player creativity. The importance of affordances for environmental interactivity and object construction is reflected in previous studies such as Ward (2015) who argues that options for personalisation of objects and environment provide opportunities for Mini C creativity as players can reflect their personal creativity in designing and customising the environment and objects. Furthermore, games that provide more freedom for the player such as open-world game designs provide a larger "possibility space" (Järvinen, 2008) which supports alternative routes of play, a greater array of strategies and opportunities for exploration.

Transferability $(M=3.8, \mathrm{SD}=.83)$ explained the greatest variance in the model (16.3\%), however, had a less positive mean score. The diversity of items included in the subscale could point to the complexity of transferability as a construct, with aspects of learning and creativity likely to transfer outside of games. The majority of items related to aspects of affective change, lending weight to previous work which has suggested games can lead to reflection on experiences (Mekler et al., 2018), new perspectives (Oliver \& Carr, 2009) and empathy (Bopp et al., 2018; Cole et al., 2015). A further two items related to specific skill development - namely problemsolving and IT/technical skills, which support previous work on the propensity of games to develop transferable skills such as problem-solving and IT literacy (e.g., Qian \& Clark, 2016; Sourmelis et al., 2017; Voulgari et al., 2014). Only one item (27.7 I have used what I have learnt from games in my job/workplace) included in the scale indicated where creativity and associated skills may be used outside of games; however, there is the possibility that respondents could have been using domain unspecific skills such as problem-solving within a vocational setting.

Despite adverts being targeted at female technology and gaming groups, the sample consisted disproportionately of men (160 males to 82 females). Western demographics between male and female gamers is roughly even with $46 \%$ of gamers in the US being female compared with 54\% being male (ESA, 2019), with the numbers being similar in Europe (ISFE, 2019). As such, future studies may wish to try and address this issue by recruiting a more equal sample of both genders and gamers from non-Western countries, thus providing a more accurate representation of a wider demographic. This would allow the CGS to be further customised to accommodate learners throughout the globe. 
Secondly, the sample was not stratified, and respondents were able to self-select. Despite the survey being advertised broadly, there is the possibility that those who completed it already self-identified as creative and as a result were more likely to take part. As has been argued elsewhere, engaging in gameplay may increase creativity and in turn, those who play games may be more creative as a result (Jackson, 2012; Jackson \& Games, 2015; Jackson et al., 2012). As such, future studies may wish to compare differences in attitudes between those who self-identify as creative and those who do not. Further confirmatory factor analysis (CFA) was not performed, and as such, future studies may wish to confirm the factor structure using a larger sample.

Chapter 12 detailed the development of a scale to measure game-specific creativity and related constructs. 71 statements on a 5-point Likert scale were subjected to PCA analysis, producing a five-factor 26-item solution with sub-scales relating to transferability, appropriation, problem-solving, affective change, and design affordances, which supported findings from a previous qualitative study. The CGS would not only benefit game developers in providing guidance on the most important aspects of player experience but could also provide benefit for educators and learners. Use of the CGS is not simply be confined to classroom-based contexts but could be used in other settings such as distance education as an alternative format of learning. Moreover, due to their ubiquitous nature, digital games could be used as an alternative method of open-world learning where learners have the autonomy to decide which games suit their preferences, and educators can use digital games as widely available educational tools. In this way, the CGS would allow educators and learners to select games that enhance the creative forms of problem-solving, appropriation and affective change and assess the development of these over time.

\subsection{Implications for practice}

Digital games are enjoyed by millions of people worldwide and offer an alternative means for developing creativity - from problem-solving abilities to affective elements such as reflection and empathy. For game developers, the CGS provides a means of measuring the effectiveness of a given game, in particular the degree to which it is facilitating player creativity. A developer could use the scale as a guide on what aspects of creativity are most important to players of a given game genre or with different previous gaming experiences and demographic characteristics. The scale could help learners and teachers capture and reflect on how creativity is developing over time and which are those experiences that are more likely to support this development.

\section{References}

Aarseth, E. (2007). I Fought the law: transgressive play and the implied player. In Situated Play, Proceedings of DiGRA 2007 Conference. Retrieved from http://www.digra.org/wpcontent/uploads/digital-library/07313.03489.pdf

Baer, J. (2016). Domain specificity: introduction and overview. In James C. Kaufman (Ed.), Domain specificity of creativity (pp. 1-16). San Diego, CA: Elsevier Ltd. 
Besemer, S., \& O'Quin, K. (1987). Creative product analysis: testing a model by developing a judging instrument. Frontiers of Creativity Research: Beyond the Basics, 367-389.

Blanco-Herrera, J. A., Gentile, D. A., \& Rokkum, J. N. (2019). Video games can increase creativity, but with caveats. Creativity Research Journal, 31(2), 119-131. doi:10.1080/1040 0419.2019 .1594524

Bopp, J.A., Opwis, K., \& Mekler, E. D. (2018). An odd kind of pleasure: differentiating emotional challenge in digital games. CHI 2018 April 21-26, Montreal QC Canada, 1-22. doi:10.1145/3173574.3173615

Burri, M. (2011). Misunderstanding creativity: user created content in virtual worlds and its constraints by code and law. International Journal of Communications Law \& Policy, 14(March), $1-28$.

Chuang, T.Y., Zhi-Feng Liu, E., \& Shiu, W.Y. (2015). Game-based creativity assessment system: the application of fuzzy theory. Multimedia Tools and Applications, 74(21), 9141-9155. doi:10.1007/s11042-014-2070-7

Cole, T., Cairns, P., \& Gillies, M. (2015). Emotional and functional challenge in core and avant-garde games. CHI Play October 3-7, 121-126. doi:10.1145/2793107.2793147

ESA. (2019). 2019 Essential facts about the computer and video game industry. Retrieved from https://www.theesa.com/wp-content/uploads/2019/05/ESA_Essential_facts_2019_ final.pdf

Gie Yong, A., \& Pearce, S. (2013). A beginner's guide to factor analysis: focusing on exploratory factor analysis. Tutorials in Quantitative Methods for Psychology, 9(2), 79-94. Retrieved from http://www.tqmp.org/RegularArticles/vol09-2/p079/p079.pdf

Gough, H. G. (1979). A creative personality scale for the adjective check list. Journal of Personality and Social Psychology, 37(8), 1398-1405. doi:10.1037/0022-3514.37.8.1398

Hall, J., Stickler, U., Herodotou, C., \& Iacovides, I. (2020a). Expressivity of creativity and creative design considerations in digital games. Computers in Human Behavior, 105. doi:10.1016/j.chb.2019.106206

Hall, J., Stickler, U., Herodotou, C., \& Iacovides, I. (2020b). Player conceptualizations of creativity in digital entertainment games. Convergence, 26(5-6), 1226-1247. doi:10.1177/ 1354856519880791

Herodotou, C., Winters, N., \& Kambouri, M. (2012). A motivationally oriented approach to understanding game appropriation. International Journal of Human-Computer Interaction, 28(1), 34-47. doi:10.1080/10447318.2011.566108

Huang,Y. H., \& Chuang, T.Y. (2012). A two-dimensional approach to creativity assessment for digital game story design. In G. Biswas, L. H. Wong, T. Hiroshima, \& W. Chen (Eds.), Proceedings of the 20th international conference on computers in education (pp. 453-458). Singapore City, Singapore: Nanyang Technological University.

Iacovides, I., Cox, A. L., Avakian, A., \& Knoll, T. (2014). Player strategies: achieving breakthroughs and progressing in single-player and cooperative games. Proceedings of the first ACM SIGCHI annual symposium on computer-human interaction in play - CHI PLAY'14. New York, 131-140. doi:10.1145/2658537.2658697

ISFE. (2019, September 12). Games in society. doi:10.3389/fnhum.2018.00047

Jackson, L. A. (2012). The upside of videogame playing. Games for Health Journal, 1(6), 452455. doi:10.1089/g4h.2012.0064

Jackson, L. A., \& Games, A. I. (2015). Video games and creativity. In G. P. Green \& J. C. Kaufman (Eds.), Video games and creativity (pp. 1-309). doi:10.1016/C2014-0-00065-2

Jackson, L. A., Witt, E. A., Games, A. I., Fitzgerald, H. E., von Eye, A., \& Zhao, Y. (2012). Information technology use and creativity: findings from the Children and Technology Project. Computers in Human Behavior, 28(2), 370-376. doi:10.1016/j.chb.2011.10.006 
Jarrett,J. (2014). Fountain hooks, emergent exploits and the playful co-creativity of MOBAs. In Videogame Cultures and the Future of Interactive Entertainment 6th Global Conference, July 19th 2014. Oxford.

Järvinen, A. (2008). Games without frontiers: theories and methods for game studies and design. University of Tampere, Finland. Retrieved from http://acta.uta.fi

Kaufman, J. C., \& Beghetto, R. A. (2009). Beyond big and little: the Four C Model of creativity. Review of General Psychology, 13(1), 1-12. doi:10.1037/a0013688

Kiili, K. (2005). Digital game-based learning: towards an experiential gaming model. Internet and Higher Education, 8(1), 13-24. doi:10.1016/j.iheduc.2004.12.001

Kirschenbaum, R. J. (1989). Understanding the creative activity ofstudents. Mansfield Center: Creative Learning Press.

Kumar, V. K., Kemmler, D., \& Holman, E. R. (1997). The creativity styles questionnairerevised. Creativity Research Journal, 10, 51-58.

Leng, E.Y., Zah, W., Ali, W., Mahmud, R. B., \& Baki, R. (2010). Computer games development experience and appreciative learning approach for creative process enhancement. Computers \& Education, 55,1131-1144. doi:10.1016/j.compedu.2010.05.011

Lubart, T. I. (1994). Creativity. In Thinking and problem solving (pp. 289-332). doi:10.1016/ B978-0-08-057299-4.50016-5

Mednick, S. A. (1968). The remote associates test. The Journal of Creative Behavior, 2(3), 213-214.

Mekler, E. D., Iacovides, I., \& Bopp, J. A. (2018). "A game that makes you question ..." Exploring the role of reflection for the player experience. In CHI PLAY'18 proceedings of the 2018 Annual symposium on computer-human interaction in play (pp.315-327). Melbourne, Australia.

Moffat, D., Crombie, W., \& Shabalina, O. (2017). Some video games can increase the player's creativity. International Journal of Game-Based Learning (IJGBL), 7(2), 35-44. doi:10.4018/ IJGBL.2017040103

Newzoo. (2020). Global Games Market Report. Accessed at: Newzoo's Global Games Market Report | Newzoo Platform.

Oliver, M., \& Carr, D. (2009). Learning in virtual worlds: using communities of practice to explain how people learn from play. British Journal of Education and Technology, 40(3), 444-457.

Qian, M., \& Clark, K. R. (2016). Game-based Learning and 21st century skills: a review of recent research. Computers in Human Behavior, 63, 50-58. doi:10.1016/j.chb.2016.05.023

Richards, R. (2007). Everyday creativity: Our hidden potential. In R. Richards (Ed.), Everyday creativity and new views of human nature: Psychological, social, and spiritual perspectives (pp. 25-53).American Psychological Association. https://doi.org/10.1037/11595-001

Runco, M. A., \& Jaeger, G. J. (2012). The standard definition of creativity. Creativity Research Journal, 24(1), 92-96. doi:10.1080/10400419.2012.650092

Shute,V.J., \& Wang, L. (2016). Assessing and supporting hard-to-measure constructs in video games. In A.A. Rupp \& J. P. Leighton (Eds.), The handbook of cognition and assessment: frameworks, methodologies, and applications. doi:10.1002/9781118956588.ch22

Sotamaa, O. (2007). On modder labour, commodification of play, and mod competitions. First Monday, 12(9), 1-12.

Sourmelis, T., Ioannou, A., \& Zaphiris, P. (2017). Massively multiplayer online role playing games (MMORPGs) and the 21st century skills: a comprehensive research review from 2010 to 2016. Computers in Human Behavior, 67, 41-48. doi:10.1016/j.chb.2016.10.020

Tabachnick, B. G., \& Fidell, L. S. (2007). Using multivariate statistics (5th ed.). Boston, MA: Allyn \& Bacon. 
Tavakol, M., \& Dennick, R. (2011). Making sense of Cronbach's alpha. International Journal of Medical Education, 2, 53-55. doi:10.5116/ijme.4dfb.8dfd

Torrance, E. P. (1966). Torrance test of creative thinking: norms and technical manual. Princeton, NJ: Personnel Press.

Torrance, E. P. (1993). The Nature of Creativity as Manifest in its Testing. In R.J. Sternberg (Eds.), The nature of creativity: contemporary psychological perspectives (pp. 43-75). Cambridge: Cambridge University Press.

Voulgari, I., Komis, V., \& Sampson, D. G. (2014). Learning outcomes and processes in massively multiplayer online games: exploring the perceptions of players. Educational Technology Research and Development, 62(2), 245-270. doi:10.1007/s11423-013-9312-7

Ward, T. B. (2015). Content, collaboration, and creativity in virtual worlds. In G. P. Green \& J. C. Kaufman (Eds.), Video games and creativity (pp. 119-136). doi:10.1016/ B978-0-12-801462-2.00006-0

Whitby, M.A., Deterding, S., \& Iacovides, I. (2019). "One of the baddies all along": moments that challenge a player's perspective. CHI PLAY 2019, 339-350. doi:10.1145/3311350. 3347192 